\title{
Transport Properties of Quantum Interference Devices the Role of the Andreev Bound States
}

\author{
Attia A. Awad Alla, A.M. Hegazy* Adel H. Phillips** \\ and Raafat K. Wassef*** \\ *Faculty of Science, Cairo University, Beni-Suef Branch, Egypt. \\ **Faculty of Engineering, Ain-Shams University, Abassia, Cairo, Egypt. \\ ***Faculty of Science, Cairo University, Giza, Egypt.
}

Quantum transport characteristic of a mesoscopic systems under the effect of Coulomb blocked and magnetic field are studied. This system is modeled as two semiconductor quantum dots which are coupled to a superconducting lead via quantum point contacts. The Josephson current density has been obtained in terms of the Andreev reflection amplitude. This Andreev reflection amplitude was deduced by solving the Bogoliubov - de Gennes (BdG) equation, describing the electron transport through the present studied junction. Numerical calculation of the obtained current density has been performed. The dependence of the current density on phase angle $\phi$ shows a periodic variation. While the oscillation features of the current density with the magnetic field are predicted to be due to quantum interference of electron waves. So such junction can be operated as a quantum interference tuner which may be valuable for nanotechnology. 


\section{Introduction:}

In recent years, the ballistic transport phenomena in mesoscopic systems have received a great deal of attention. With device dimensions smaller than the electron phase coherence length as well as the elastic mean free path, the scattering and propagation of electrons are described by phase - coherent wave function.

The electronic stub tuner, a quantum interference device, has been studied by many authors (1-9). A conductance oscillations were observed The results were explained as the quantum interference of electronic waves propagating along the wire and the stub.

In the present paper, a model was proposed as two semiconductor quantum dots coupled to a superconducting lead via quantum point contacts. The quantum transport characteristics of this model are studied under the effect of coulomb blockade and a magnetic field. The present device has not been studied previously.

\section{Theoretical Treatment and the Model}

In this model we shall study the transport properties of a quantum interface device, i.e. the electronic stub tuner under the effect of the magnetic field, B,. This model is schematically represented in Fig. (1) as the S-Sm-S-Sm$\mathrm{S}$ (S-superconductor, Sm-semiconductor ). The dc Josephson current is calculated by solving the Bogoluibov-de Gennes equation (BdG) [10].

$$
\left(\begin{array}{cc}
\mathrm{H} & \Delta \\
\Delta^{*} & -\mathrm{H}
\end{array}\right) \psi(\mathrm{x})=\mathrm{E} \psi(\mathrm{x})
$$

The pair potential $\Delta$ in this case is given by

$$
\Delta(\mathrm{x})= \begin{cases}\Delta_{0} \exp (\mathrm{i} \varphi) & , \mathrm{x}<0 \\ 0 & , 0<\mathrm{x}<\mathrm{L} \\ \Delta_{0} \exp (\mathrm{i} \varphi) & , \mathrm{L}<\mathrm{x}<2 \mathrm{~L} \\ 0 & , 2 \mathrm{~L}<\mathrm{x}<3 \mathrm{~L} \\ \Delta_{0} \exp (\mathrm{i} \varphi) & , \mathrm{x}>3 \mathrm{~L}\end{cases}
$$

where $\Delta_{0}$ is the energy gap of the superconductor, $\phi$ is the phase difference of the Cooper pair and L is the distance between the two successive superconducting reservoir's. The Hamiltonian, $\mathrm{H}$ for this model is expressed as 


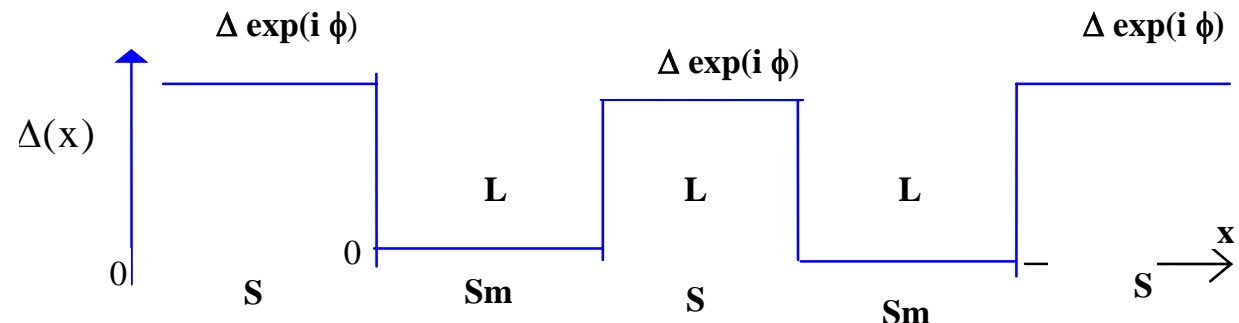

Fig.(1) Schematic representation of a S-SıII-د-Sm-S jukn.... with step wise pair potential.

$$
\mathrm{H}=-\frac{\hbar^{2} \mathrm{~d}^{2}}{2 \mathrm{~m}_{\mathrm{dx}}{ }^{2}}-\frac{\mathrm{j}^{2} \pi^{2}}{\mathrm{~b}^{2}}+\mathrm{V}_{0}-\mathrm{E}_{\mathrm{F}}+\frac{\mathrm{U}_{\mathrm{C}} \mathrm{N}^{2}}{2}+e \eta \mathrm{V}_{\mathrm{g}} \mathrm{N}+\mathrm{S}+\frac{\hbar \mathrm{eB}}{2 \mathrm{~m}}
$$

In Eq.(3), b ,is the width of the semiconductor stub.

There are two kinds of bound states, according to the processes that set up the bound states. The first, called the p-process [11], is constructed from eigenstates having superscript $(+)$, which is associated with right going electronlike quasiparticles in the semiconductor regions. The second called n-process, is constructed from eigenstates having superscript (-) and is associated with right-going holelike quasiparticles in the semiconductor regions. Now, accordingly, the p-process eigenfunction is given by[12]

$$
\Psi(\mathrm{x})= \begin{cases}\left(\mathrm{a}_{\Psi_{\mathrm{S}, \mathrm{h}}^{+}}^{+} /\left(\left.\mathrm{u}_{0}\right|^{2}+\left|\mathrm{v}_{0}\right|^{2}\right)^{0.5}\right. & , \text { region(I) } \\ \mathrm{C}_{1} \Psi_{\mathrm{Sm}, \mathrm{e}}^{+}+\mathrm{D}_{1} \Psi_{\mathrm{Smh}}^{+} & , \text {region(II) } \\ \left(\mathrm{C} \Psi_{\mathrm{S}, \mathrm{e}}^{+}+\mathrm{d}_{\Psi_{\mathrm{S}, \mathrm{h}}^{+}}^{+} /\left(\left|\mathrm{u}_{0}\right|^{2}+\left|\mathrm{v}_{0}\right|^{2}\right)^{0.5}\right. & , \text { region(III) } \\ \mathrm{C}_{2}^{+} \Psi_{\mathrm{Sme}}^{+}+\mathrm{D}_{2} \Psi_{\mathrm{Smh}}^{+} & , \text {region(IV) } \\ \left(\mathrm{b} \Psi_{\mathrm{S}, \mathrm{e}}^{+}\right) /\left(\left.\mathrm{u}_{0}\right|^{2}+\left|\mathrm{v}_{0}\right|^{2}\right)^{0.5} & , \text { region(V) }\end{cases}
$$

The subscript e (h) indicates the electronlike (holelike)quasiparticle. $\mathrm{u}_{0}$ and $\mathrm{v}_{0}$ in Eq.(4) is given by Eq 


$$
u_{0}=\sqrt{\frac{1}{2}\left(1+\frac{\left(E^{2}-\Delta_{0}^{2}\right)^{1 / 2}}{E}\right)} \quad, \quad v_{0}=\sqrt{\frac{1}{2}\left(1-\frac{\left(E^{2}-\Delta_{0}^{2}\right)^{1 / 2}}{E}\right.}
$$

The n-process eigenfunction can be obtained from Eq.(4) by changing the superscript (+) to (-) and the subscript (e,h) to (h,e). In Eq.(4) normalized eigenfunctions in the semiconductor regions are expressed as

$$
\begin{aligned}
& \Psi_{S m, e}^{ \pm}(x)=\left(\begin{array}{l}
1 \\
0
\end{array}\right) \exp \left( \pm i \underset{k_{e}}{\sim} x\right) \\
& \Psi_{S m, h}^{ \pm}(x)=\left(\begin{array}{l}
0 \\
1
\end{array}\right) \exp \left( \pm \dot{i}_{k_{h}}^{\sim} x\right)
\end{aligned}
$$

Where the parameter $k_{e, h}$ is given by

$$
\tilde{\mathrm{k}_{\mathrm{e}, \mathrm{h}}}=\left(\frac{2 \mathrm{~m}}{\hbar^{2}}\right)^{0.5}\left[\mathrm{U}_{ \pm}+\sigma^{\mathrm{e}, \mathrm{h}} \mathrm{E}-\left(\frac{\mathrm{j} \pi}{\mathrm{b}}\right)^{2}\right]^{0.5}
$$

Where $\sigma^{e}=1$ for electronlike quasiparticle, $\sigma^{h}=-1$ for holelike quasiparticle.

and the normalized eigenfunctions in the superconductor regions are given by

$$
\begin{aligned}
& \Psi_{s, e}^{ \pm}=\left(\begin{array}{l}
u_{0} \exp (i \varphi) \\
v_{0}
\end{array}\right) \exp \left( \pm i_{k_{e}} x\right) \\
& \Psi_{s, h}^{ \pm}=\left(\begin{array}{l}
v_{0} \exp (i \varphi) \\
u_{0}
\end{array}\right) \exp \left( \pm i_{k_{h}} x\right)
\end{aligned}
$$

where $\mathrm{k}_{\mathrm{e}, \mathrm{h}}$ is given by

$$
\mathrm{k}_{\mathrm{e}, \mathrm{h}}=\left(\frac{2 \mathrm{~m}}{\hbar^{2}}\right)^{0.5}\left[\mathrm{U}_{ \pm}+\sigma^{\mathrm{e}, \mathrm{h}}\left(\mathrm{E}^{2}-\Delta_{0}^{2}\right)^{0.5}-\left(\frac{\mathrm{j} \pi}{\mathrm{b}}\right)^{2}\right]^{0.5}
$$

in which

$$
\mathrm{U}_{ \pm}=\frac{\mathrm{U}_{\mathrm{C}} \mathrm{N}^{2}}{2}+\mathrm{e \eta} \mathrm{V}_{\mathrm{g}} \mathrm{N}+\mathrm{S}+\frac{\hbar \mathrm{eB}}{2 \mathrm{~m}}+\mathrm{V}_{0} \pm \mathrm{E}_{\mathrm{F}}
$$

The current density, $\mathbf{J}$, due to the Andreev levels can be calculated by using the following Eq.[13] 


$$
\begin{aligned}
& \mathrm{J}=\left(\frac{\mathrm{e} \hbar}{\mathrm{m}}\right) \sum_{\alpha= \pm 1} \alpha_{\mathrm{k}_{\mathrm{e}, h}}^{ \pm}\left[\tanh \left(\mathrm{E} / 2 \mathrm{k}_{\mathrm{B}} \mathrm{T}\right)\right]^{2}\left(\frac{\mathrm{e}^{2}}{\Delta_{0} \mathrm{~L}}\right)^{0.25} \\
& \left\{\mathrm{f}_{\mathrm{FD}}\left(\mathrm{U}-\mathrm{V}_{0}\right)-\mathrm{f}_{\mathrm{FD}}(\mathrm{U})\right\}\left[\frac{\Gamma_{\mathrm{p}} \Gamma_{\mathrm{n}}}{\Gamma_{\mathrm{p}}+\Gamma_{\mathrm{n}}}\right]
\end{aligned}
$$

where $\Gamma_{p / n}=\left|a\left(E_{\alpha}, \alpha \varphi\right)\right|^{2}$ is the Andreev scattering probability, and $\alpha=+1$ $(-1)$ refers to the p-process and (n-process), and $f_{F D}$ is the Fermi-Dirac distribution function.

The Andreev scattering probability for both, p-process and n-process are obtained after matching the eigenfunctions (Eq.(6a,b , 8a,b) at all S-Sm interfaces, we get

$$
\begin{aligned}
& \Gamma_{p}=\left.a\left(E_{+},+\varphi\right)\right|^{2}
\end{aligned}
$$

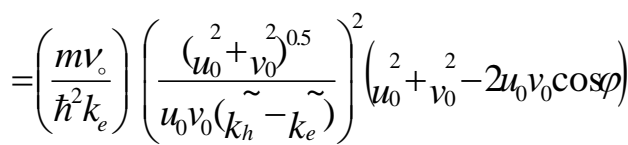

$$
\begin{aligned}
& \Gamma_{n}=\left.a\left(E_{-},-\varphi\right)\right|^{2} \\
& =\left(\frac{m V_{0}}{\hbar^{2} k_{h}}\right)\left(\frac{\left(u_{0}^{2}+v_{0}^{2}\right)^{0.5}}{u_{0} v_{0}\left(\tilde{k_{e}}-\tilde{k_{h}}\right)}\right)^{2}\left({ }_{u_{0}}^{2}+v_{v_{0}}^{2}-2 u_{0} v_{0} \cos \varphi\right)
\end{aligned}
$$

Substituting Eq. (12,13) in Eq.(11)

$$
\begin{aligned}
& \mathrm{J}=\left(\frac{\mathrm{e} \hbar}{\mathrm{m}}\right) \sum_{\mathrm{j}}\left(\frac{\mathrm{mV_{0 }}}{2 \hbar^{2}}\right)\left[\operatorname{tank}\left(\frac{\mathrm{E}}{2 \mathrm{k}_{\mathrm{B}} \mathrm{T}}\right)\right]^{2}\left[\frac{1}{\left(\mathrm{ke}^{\tilde{2}-\mathrm{kh}}\right)}\right]^{2} \\
& \left.\left\{1-\left[1-\frac{\left(\mathrm{E}^{2}-\Delta_{0}^{2}\right)^{0.5}}{\mathrm{E}^{2}}\right]^{0.5} \cos (\varphi)\right\}\left\{41-\frac{\left(\mathrm{E}^{2}-\Delta_{0}^{2}\right)^{0.5}}{\mathrm{E}^{2}}\right]^{-1}\right\} \\
& {\left[\frac{\mathrm{e}^{2}}{\Delta_{0} \mathrm{~L}}\right]^{0.25}\left\{\mathrm{f}_{\mathrm{FD}}\left(\mathrm{U}-\mathrm{V}_{0}\right)-\mathrm{f}_{\mathrm{FD}}(\mathrm{U})\right\}}
\end{aligned}
$$

The Josephson current density, J, equation (14) depends on the following parameters: 
1- the phase difference $\phi$ of the Cooper pair,

2- the width of the semiconductor quantum dot, b,

3- the distance, $\mathrm{L}$, between the two superconducting reservoirs,

4- the gate voltage, $V_{g}$,

5- the energy gap of the superconductor, $\Delta_{0}$,

6- the magnetic field, $\mathrm{B}$,

7- the Schottky barrier height, $\mathrm{S}$,

8- the Coulomb energy, $\mathrm{U}_{\mathrm{C}}$, for the number of the electrons $(\mathrm{N}+1)$ and $\mathrm{N}$ in the quantum dot,

These above mentioned parameters can be varied and controlled in order to fabricate the desired junction for optimum utility in microelectronics technology.

\section{Numerical Calculation and Results:}

In order to show the reliability of the present theoretical treatment for the present model of superconductor-semiconductor junction, we have performed a numerical calculation. The electron transport through the junction is treated as a stochastic process, so that the tunneled electron energy as a random number. Also, the Schottky barrier height, $\mathrm{S}$, is determined by using the Monte-Carlo simulation technique and its value was found to be $\sim 0.47 \mathrm{eV}$ for the case $\mathrm{Nb}-\mathrm{GaAs} \mathrm{Nb-GaAs}$ based heterostructure interface. This value of, $\mathrm{S}$ was found in agreement with those found experimentally [14] and by us [ 1518]. Also, the number of electrons in the quantum dot is computed by using the Monte-Carlo simulation technique.

1. The variation of the current density, J, with temperature (Fig.2) shows that the decreases of the current as the temperature increases. This result show a qualitative agreement with those published in the literature [19-21]

2. Fig.s (3) show the variation of the current density, J, with, L, for different values of, $\phi, T, B$. Also Fig.s (4) show the variation of, J, with, b, under different values of $\phi, T, B$. It may be noted that the current density, J, becomes approximately constant where, L, attains a value $\sim 80 \mathrm{~nm}$. This value was found to be equal to Fermi wavelength $\lambda_{\mathrm{F}}$, for mesoscopic system [22]. But for the case of, b, the current density, J, becomes constant when , b, attains a value $\sim 10 \mathrm{~nm}$. This value does not change as the temperature varies. However, the value of, J, does not change when the values of $\phi$, and B, vary. 


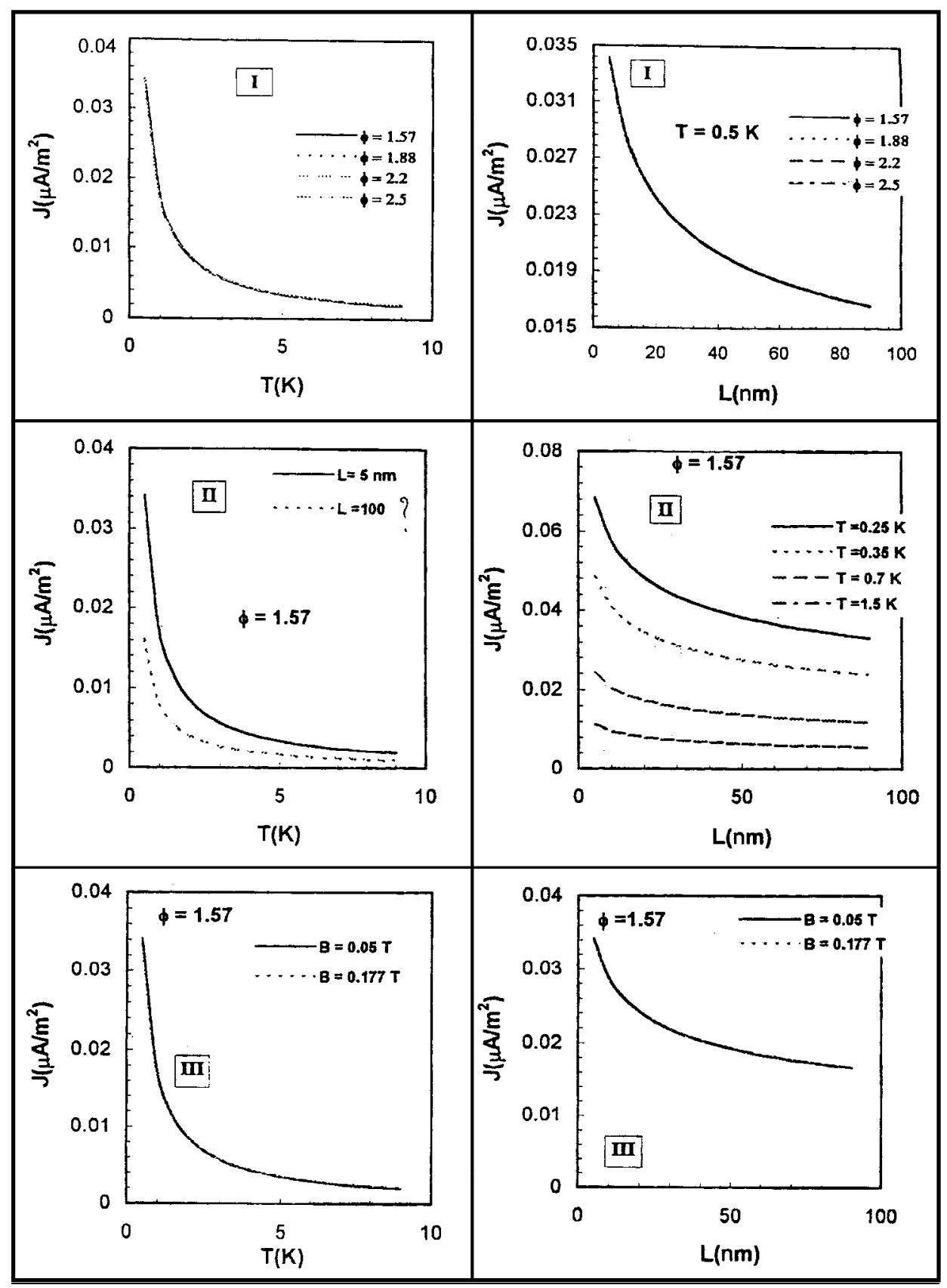

Fig.(2) The current density as a function of temperature relation for different Values of $\phi(\mathrm{I}), \mathrm{L}(\mathrm{II})$, and B(III).

Fig.(3) The current density as a function of length of the semiconductor quantum dot relation for different values of $\varphi(\mathrm{I}), \mathrm{T}(\mathrm{II})$,and B(III). 


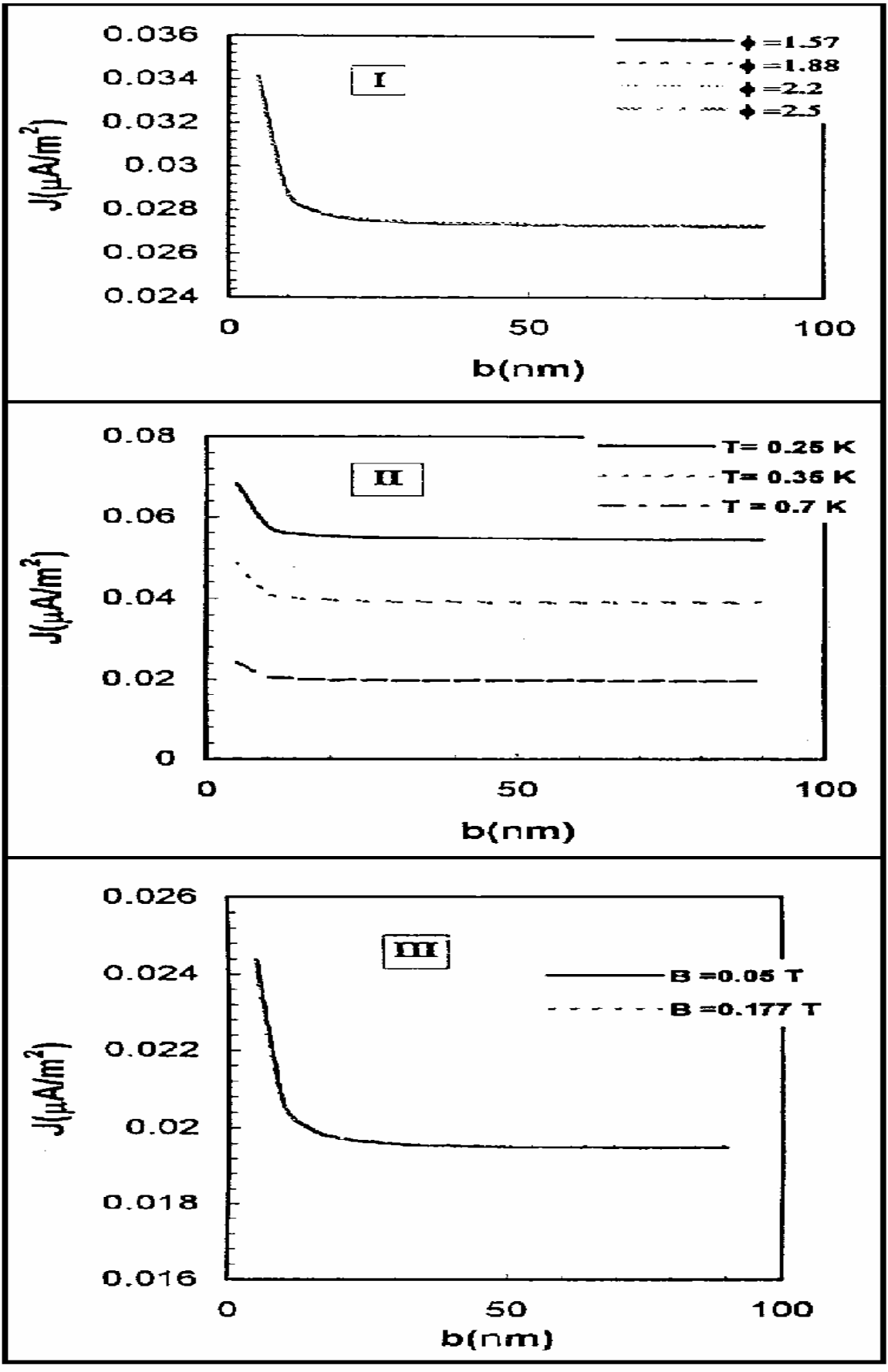

Fig.(4) The current density as a function of the width of the semiconductor quantum dot relation for different values of $\varphi(\mathrm{I}), \mathrm{T}(\mathrm{II})$, and B(III). 
3. A periodic variation of, J, with $\phi$ is shown in Fig.(5). This result was observed by another authors $[13,23]$ previously which shows the coherent property of the system and it is in a clean limit.

4. The variation of the current density, J, with the magnetic field, B, (Fig.6) show a resonance behavior at the same values of the magnetic field, $\mathrm{B}$. The oscillatory behavior was observed previously $[9,24]$ and this is due to quantum interference of electron under the effect of the magnetic field B.
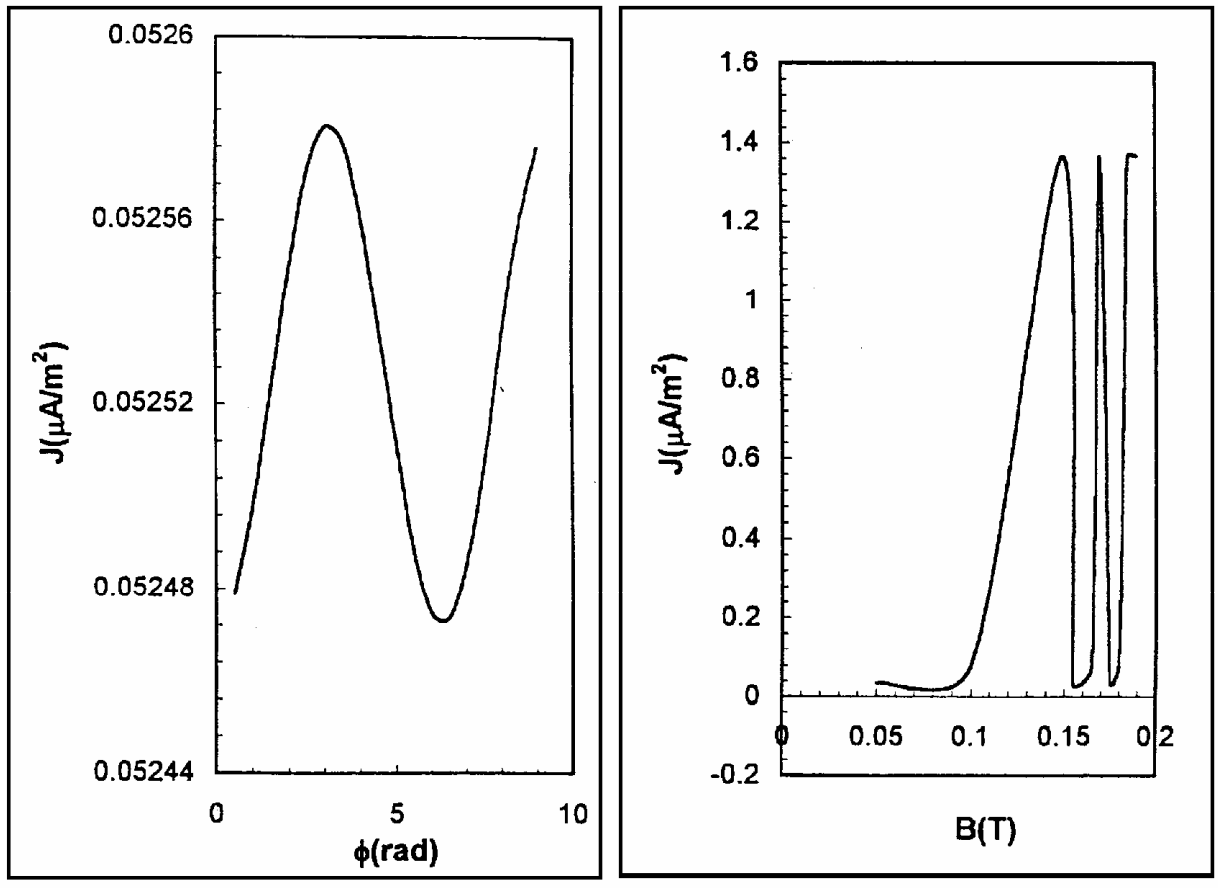

Fig.(5) The variation of the current density with phase angle.

Fig.(6) The variation of current density as a function of magnetic field.

\section{Conclusion:}

In this paper we derived a josephson current density for the proposed device in terms of the Andreev reflection amplitude. The oscillatory behavior of the current density with the magnetic field is explained as the quantum interference of electron waves. We can conclude that such mesoscopic structure used as quantum devices, are predicted to be operational at very high frequencies. 


\section{Reference:}

1. F. Sols, M. Macucci, U. Ravaioli, and K. Hess, Appl. Phys. Lett. 54, 350 (1989); J. Appl. Phys. 66, 3892 (1989).

2. S. Datta, Superlattices microstruct. 6, 83 (1989).

3. D.C. Miller, R.K. Lake, S. Datta, M.S. Lundstrom, M.R. Melloch, and R. Reifenbenberger, Nanostructure Physics and Fabriction, edited by M.A. Reed and W.P. Kirk (Academic, new York 1989).

4. K. Aihara, M. Yamamoto, and T. Mizutani, J. App. Phys. 31, L916 (1992); Appl. Phys. Lett. 63, 3595 (1993).

5. R. Aakis, P. Vasilopoulos, and P. Debray, Phys. Rev. B 52 (1995)2805.

6. A. Weisshaar, J. Lary, S.M. Goodnick, and V.K. Tripathi, Appl. Phys. Lett. 55, 2114 (1989)

7. Y.S. Joe, M. Khatun, and R.M. Cosby, Solid State Commun., 93, 943 (1995).

8. Y.S. Joe, M. Khatun, and R.M. Cosby, J. Appl. Phys. 78, 34409 (1998).

9. M. Khatun, P. K. Joyner, R. M. Cosby, and Y. S. Joe, J. Appl. Phys. 84, 34409 (1998).

10. P.G. de Gennes, 1966, Superconductivity of metals and Alloys (Benijamin, New York).

11. S. Kashiway, Y. Tanaka, M. Koyanagi, and K. Kajimura, Jpn. J. Appl. Phys. 34, 4555 (1995).

12. L. F. Chang and P.F. Bagwell, Phys. Rev. B 49, 15853 (1994).

13. V.C.Y. Chang and C.S. Chu, Phys. Rev. B 55, 6004 (1997).

14. Th.Becker, M.Muck, and Ch. Heidenet, Physica B 204, 183 (1995)

15. A.H.Aly, Adel H. Phillips and R.K. Wassef, Egyptian J. of Physics 70, 35 (1998).

16. Aziz N. Mina, Adel H. Phillips, F.M.Shaheen and N.S. Abdel Gawad, accepted for publication in physica $C$, special issue of conference $6^{\text {th }}$ International conference, Materials and Mechanisms of superconductivity and High Temperature superconductors February (20-25), 2000 George R. Brown convention Center and Hyatt Regency Hotel-Downtown Houston State University, Texas, U.S.A

17. A.A. Awad Alla, A.M. Hegazy, A.H. Phillips and Raafat K. Wassef, accepted in Egyptian Journal of Physics (1999) and presented in $6^{\text {th }}$ International conference, Materials and Mechanisms of superconductivity and High Temperature superconductors February (20-25), 2000, George R. Brown convention Center and Hyatt Regency Hotel-Downtown Houston State University, Texas, U.S.A.

18. A.A. Awad Alla, A.M. Hegazy, A.H. Phillips and Raafat K. Wassef, presented in $6^{\text {th }}$ International conference, Materials and Mechanisms of 
superconductivity and High Temperature superconductors February (20-25), 2000, George R. Brown convention Center and Hyatt Regency Hotel-Downtown Houston state University, Texas, U.S.A.

19. T.M. Klapwijk, Physica B 197, 481 (1994).

20. J. Nitta, T. Akazaki and H. Takayangi, Phys. Rev. B 46, 14286 (1992).

21. W.M. van Hufflen, D.R. Heslinga, M.J. deBoer, and N. van der Post, Phys. Rev. B 47, 5170 (1993).

22. G. Smith, Rep. Prog .Phys. 235, 235 (1996).

23. H.van Houten and C.W.J.Beenakker, Physica, B 175, 187 (1991).

24. G.S.Vieria, P.S.S. Guimaraes, E.S Alves, S.J. Allen, K.L. Campman, and A.C.Gossarad, Physica B 256 -258, 527 (1998). 